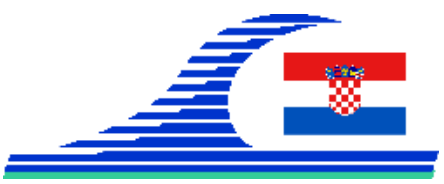

\title{
Influence of composition parameters on the tunnel lining concrete made with dredged sediments subjected to high temperatures
}

\author{
Fatiha KAZI AOUAL-BENSLAFA ${ }^{1}$, Mohammed AMEUR ${ }^{1}$, \\ Belkacem MEKERTA ${ }^{1}$, Abdelaziz SEMCHA ${ }^{1}$
}

\author{
1. Laboratoire LabMat, ENP Oran, BP 1523, Oran El Ménaouer, 31000 Oran, Algeria \\ fkaziaoual@yahoo.fr ; mohammed.ameur@gmail.com; mekertab@yahoo.fr ; \\ asemcha@yahoo.fr
}

\section{Résumé :}

Le développement de filières de valorisation des sédiments de dragage (SD) du port d'Oran est une nécessité pour palier problème environnemental majeur causé par leur largage en mer. Cette étude expérimentale a été réalisée pour étudier l’influence des SD sur le comportement du béton en conditions extrêmes de températures pour une application précise : le revêtement de tunnel. A cet effet, deux bétons ont été fabriqués ; B1 fabriqué avec des granulats terrestres et B2 avec des SD. L'influence du remplacement du sable fin par des SD sur la résistance en compression en conditions normales à $20^{\circ} \mathrm{C} \mathrm{Rc}_{20}$ et sur les résistances résiduelles en compression $\mathrm{R}_{\mathrm{CR}}$ après chauffage à des températures de $150^{\circ} \mathrm{C}, 350^{\circ} \mathrm{C}$ et $600^{\circ} \mathrm{C}$ et refroidissement à l'air et dans l'eau, a été étudiée. Les résultats ont montré l'effet bénéfique de la substitution du sable fin par les SD sur la $\mathrm{Rc}_{20}$ du béton $\mathrm{B} 2$ qui a été légèrement améliorée. Les effets des hautes températures sur la $\mathrm{R}_{\mathrm{CR}}$ des bétons sont plus accentués lorsque les bétons sont soumis à un choc thermique dans l'eau que dans l'air.

Mots-clés : Béton, Revêtement de tunnel, Sédiments de dragage, Haute température, Refroidissement air et eau, Résistance en compression.

\section{Introduction}

Le curage de fond du port d'Oran est une opération d'entretien indispensable au développement de l'activité économique. Se pose alors la question du devenir des sédiments dragués à laquelle il convient d'apporter des solutions respectueuses de l'environnement. Des stratégies avec une vision de développement durable ont été développées pour l'utilisation bénéfique des SD dans la construction de routes (BEN ABDELGHANI et al., 2014), dans la fabrication de briques (GAO et al., 2017) et dans les mortiers et bétons (KAZI AOUAL-BENSLAFA et al., 2015 ; ZAMBON et al., 2015). Le béton est un matériau régional, il est largement utilisé dans les structures de revêtement des tunnels mais doit répondre à des exigences de performances mécaniques 
Mediterranean rocky coasts:

Features, processes, evolution and problems

et de durabilité. Les inspections des tunnels après des incendies majeurs (PIARC-AIPCR, 2007) et les études sur l'endommagement des bétons soumis à de hautes températures, ont mis en évidence que les performances résiduelles des bétons sont très faibles. Des études ont été réalisées sur l'effet des régimes de refroidissement du béton fabriqués avec différents granulats (LUO et al., 2000 ; KAZI AOUAL-BENSLAFA et al., 2011). Les études entamées depuis plusieurs années au laboratoire LabMat ont permis la caractérisation complète des SD (KAZI AOUAL-BENSLAFA et al., 2014) et leur utilisation dans les mortiers (KAZI AOUAL-BENSLAFA et al., 2015). A ce jour, le comportement des bétons fabriqués avec des SD en conditions extrêmes de température est mal établi et des travaux de recherche sont nécessaires. L’intérêt du travail expérimental présenté est de fournir des données sur les propriétés mécaniques résiduelles des bétons fabriqués avec des SD après échauffement et refroidissement à l'air et dans l'eau.

\section{Matériaux et méthodes}

\subsection{Matériaux}

Le béton est un mélange de ciment, de granulats et d'eau dans lequel des ajouts peuvent être incorporés. Le ciment utilisé est un ciment CPJ CEMII/A 42,5 de la cimenterie d'Ogaz-Sig dans l'Ouest Algérien. Il a un poids volumique de $3100 \mathrm{~kg} / \mathrm{m}^{3}$ et une surface spécifique Blaine égale à $358.5 \mathrm{~m}^{2} / \mathrm{kg}$.

Les granulats terrestres utilisés proviennent de la carrière de Kristel (Oran Est), ils sont de nature calcaire. Le sable $0 / 4 \mathrm{~mm}$ est un mélange de sable grossier corrigé par du sable fin de Terga. Les graviers sont des granulats concassés avec un diamètre maximal de $16 \mathrm{~mm}$. Les sédiments de dragage (SD) utilisés ont été collectés au port d'Oran. Les caractéristiques physiques et chimiques sont données au tableau 1. Les SD contiennent 6\% d'argiles, 39\% de limons et 55\% de sables. Les courbes granulométriques des différents granulats et des SD sont données en figure 1.

\subsection{Les méthodes}

La composition du béton a été formulée selon la norme Européenne 206-1. Des essais préliminaires ont été réalisés afin d'optimiser la composition du béton. Le tableau 2 donne un résumé de la composition des deux bétons. Les essais de température ont été réalisés suivant la norme Européenne (EN 192-1-2, 2004). Quatorze séries d'éprouvettes de béton $\left(100 \times 100 \times 100 \mathrm{~mm}^{3}\right)$ ont été confectionnées, démoulées après 24h et mises à la cure dans l'eau pendant 28jours et dans l'air jusqu'à l'échéance de 150jours. Les bétons confectionnés sont nommés $\mathrm{B} 1_{20}, \mathrm{~B} 2_{20}$ (bétons non chauffés, $20^{\circ} \mathrm{C}$ ), B $1_{150}, \mathrm{~B} 2_{150}$ (bétons chauffés à $150^{\circ} \mathrm{C}$ ), $\mathrm{B} 1_{350}, \mathrm{~B} 2_{350}$ (bétons chauffés à $350^{\circ} \mathrm{C}$ ), $\mathrm{B} 1_{600}, \mathrm{~B} 2_{600}$ (bétons chauffés à $600^{\circ} \mathrm{C}$ ). Les éprouvettes sont ensuite séchés dans une étuve à une température de $40^{\circ} \mathrm{C}$ jusqu'à la stabilisation de leur poids. A la fin de cette 
Mediterranean rocky coasts:

Features, processes, evolution and problems

étape, elles sont chauffées à des températures de $150^{\circ} \mathrm{C}, 350^{\circ} \mathrm{C}$ et $600^{\circ} \mathrm{C}$, dans un four à moufles avec une vitesse de chauffe de $10^{\circ} \mathrm{C} / \mathrm{mn}$, maintenues à la température pendant une heure et ensuite le refroidissement du four est programmé avec la même vitesse. Une fois retirées du four, 6 séries d'éprouvettes sont refroidies à l’air et ensuite écrasées pour la détermination des résistances résiduelles en compression. Les 6 autres séries d'éprouvettes sont refroidies à l'eau ensuite écrasées pour la détermination des résistances résiduelles en compression. Le tableau 3 résume le traitement des différentes séries de bétons fabriqués.

Tableau 1. Caractéristiques physiques et chimiques des SD (KAZI AOUAL- BENSLAFA et al., 2014).

\begin{tabular}{ll|ll}
\hline \multirow{2}{*}{ Caractéristiques physiques } & \multirow{2}{*}{ Valeur mesurée } & $\begin{array}{l}\text { Composants chimiques } \\
\text { (\%) }\end{array}$ & Teneur (\%) \\
\hline Teneur en eau (\%) & 63.5 & $\mathrm{SiO}_{2}$ & 54.80 \\
\hline \multirow{2}{*}{ Surface specifique Blaine $\left(\mathrm{m}^{2} / \mathrm{kg}\right)$} & \multirow{2}{*}{992} & $\mathrm{CaCO}_{3}$ & 19.30 \\
\cline { 3 - 4 } & \multirow{2}{*}{2450} & $\mathrm{Al}_{2} \mathrm{O}_{3}$ & 4.30 \\
\cline { 3 - 4 } & & $\mathrm{Fe}_{2} \mathrm{O}_{3}$ & 0.44 \\
\hline \multirow{2}{*}{ Poids volumique $\left(\mathrm{kg} / \mathrm{m}^{3}\right)$} & \multirow{2}{*}{24.7} & $\mathrm{SO}_{3}$ & 0.00 \\
\cline { 3 - 4 } & & $\mathrm{CO}_{2}$ & 0.00 \\
\hline pH & 8.8 & $\mathrm{H}_{2} \mathrm{O}$ & 6.62 \\
\hline Bleu de méthylène & 4.8 & Carbonates & 40.5 \\
\hline Conductivité électrique $(\mathrm{dS} / \mathrm{m})$ & 0.9 & Matière organique & 8.1 \\
\hline
\end{tabular}

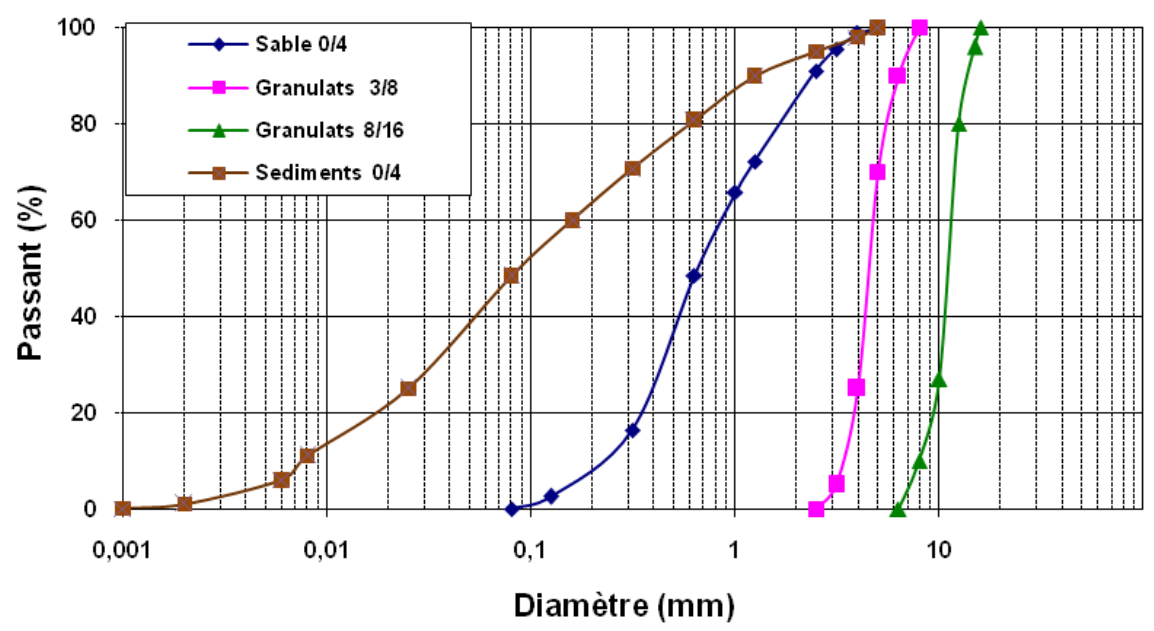

Figure 1. Les courbes granulométriques des différents granulats et des SD.

Tableau 2. Composition des bétons.

\begin{tabular}{lll}
\hline Composants & B1 & B2 \\
\hline Ciment $(\mathrm{kg})$ & 475 & 475 \\
\hline Graviers $(3 / 8+8 / 16)(\mathrm{kg})$ & 1115 & 1115 \\
\hline Sable $(\mathrm{kg})$ & 656 & 492 \\
\hline Sédiments de dragage $(\mathrm{kg})$ & 0 & 164 \\
\hline Eau $(\mathrm{L})$ & 214 & 214 \\
\hline
\end{tabular}


Mediterranean rocky coasts:

Features, processes, evolution and problems

Tableau 3. Nombre et type d'éprouvettes de béton.

\begin{tabular}{llll}
\hline \multicolumn{4}{c}{28 séries d'éprouvettes cubiques $\left(\mathbf{1 0 0 \times 1 0 0 \times 1 0 0 \mathbf { m m } ^ { 3 } )}\right.$} \\
\hline $20^{\circ} \mathrm{C}$ & $150^{\circ} \mathrm{C}$ & $350^{\circ} \mathrm{C}$ & $600^{\circ} \mathrm{C}$ \\
\hline \multirow{2}{*}{2 séries } & 2 séries refroidies à l'air & 2 séries refroidies à l'air & 2 séries refroidies à l'air \\
\cline { 2 - 4 } & 2 séries refroidies dans l'eau & 2 séries refroidies dans l'eau & 2 séries refroidies dans l'eau \\
\hline
\end{tabular}

\section{Résultats et discussion}

\subsection{Le comportement des bétons à l'état durci à $20^{\circ} \mathrm{C}$}

Les résultats de la résistance à la compression à la température de $20^{\circ} \mathrm{C}\left(\mathrm{Rc}_{20}\right)$ sont donnés en figure 2. On remarque que la $\mathrm{Rc}_{20}$ à 7 et 28 jours du béton $\mathrm{B} 2$ fabriqué avec les sédiments de dragage est similaire à celle du B1 fabriqué avec les granulats terrestres alors qu'elle est améliorée aux échéances de 60 et 90jours. L'augmentation de la teneur en agrégats fins est responsable de l'augmentation légère de la résistance Rc des bétons. Les résultats similaires ont été trouvés par LIMEIRA et al., (2011). A cet effet nous concluons que le sable fin de Terga peut être remplacé par les sédiments de dragage du port d'Oran dans la composition du béton. Cette substitution a un double impact, le premier est la préservation de l'environnement et du pillage du sable de la côte de Terga et le second est la valorisation des sédiments de dragage.

\subsection{Les résistances résiduelles à la compression des bétons}

Les résistances résiduelles à la compression des bétons chauffés à différentes températures et soumis à des chocs thermiques dans l'eau et à l'air ont été mesurées. Les résultats sont donnés en figure 3 sous forme comparative pour les deux régimes de refroidissement air et eau. On note une perte de résistance de tous les bétons qui s’accentue avec la température.

Après échauffement des bétons et refroidissement à l'air

A la température $150^{\circ} \mathrm{C}$, la résistance $\mathrm{Rcr}$ du béton B1air représente $78 \%$ de la $\mathrm{Rc}_{20}$ et que la résistance $\mathrm{Rcr}$ du béton B2air représente $80 \%$ de la $\mathrm{Rc}_{20}$. A la température $350^{\circ} \mathrm{C}$, la résistance $\mathrm{Rcr}$ du béton B1air représente $74 \%$ de la $\mathrm{Rc}_{20}$ et que la résistance $\mathrm{Rcr}$ du béton B2air représente $78 \%$ de la $\mathrm{Rc}_{20}$. A la température $600^{\circ} \mathrm{C}$, la résistance $\mathrm{Rcr} d u$ béton $\mathrm{B} 1$ air représente $30 \%$ de la $\mathrm{Rc}_{20}$ et que la résistance $\mathrm{Rcr}$ du béton B2air représente $32 \%$ de la $\mathrm{Rc}_{20}$.

Après échauffement et refroidissement à l'eau

A la température $150^{\circ} \mathrm{C}$, la résistance Rcr des bétons B1eau B2eau représente $98 \%$ de la $\mathrm{Rc}_{20}$. A la température $350^{\circ} \mathrm{C}$, la résistance $\mathrm{Rcr}$ des bétons $\mathrm{B} 1$ eau et $\mathrm{B} 2$ eau représente $90 \%$ de la $\mathrm{Rc}_{20}$. A la température $600^{\circ} \mathrm{C}$, la résistance Rcr des bétons B1eau et B2eau représente $31 \%$ de la $\mathrm{Rc}_{20}$. 
Mediterranean rocky coasts:

A la lecture des résultats on constate qu'à la température de $150^{\circ} \mathrm{C}$ la résistance $\mathrm{Rcr}$ des bétons refroidis à l'air, diminue alors que celle des bétons refroidis à l'eau est beaucoup plus élevée. Ces résultats sont semblables à ceux trouvés ailleurs. Les chercheurs conviennent que la déshydratation du béton pendant le chauffage est à l'origine de la diminution de sa résistance résiduelle. À une température inférieure à $150^{\circ} \mathrm{C}$, l'eau libre s'évapore, les bétons chauffés à $150^{\circ} \mathrm{C}$ et refroidis à l'eau sont réhydratés et une grande partie de l'eau libre évaporée est récupérée. Entre les températures de $150^{\circ} \mathrm{C}$ et $350^{\circ} \mathrm{C}$, l'eau liée contribue à l'établissement des pressions internes agissant comme force de précontrainte qui donne le pic de résistance. La réduction de la valeur de pointe dans les bétons B1air350, B2air350 est attribuée à une réduction de la pression interne pendant le refroidissement à l'air. Ceci est dû au phénomène de transfert d'une partie de la vapeur d'eau des zones chaudes du noyau de l'éprouvette aux zones extérieures refroidies. Au-delà de $350^{\circ} \mathrm{C}$, on observe une diminution quasi linéaire et importante de la résistance résiduelle avec un gradient plus prononcé dans les bétons soumis à des chocs thermiques dans l'eau. Tous les bétons chauffés à une température de $600^{\circ} \mathrm{C}$, ont le même comportement mécanique résiduel. De nombreux chercheurs (LUO et al., 2000 ; ZHANG, 2011) ont émis des observations similaires. Probablement cela est dû au fait que toute l'eau dans le béton a disparu à cette température.

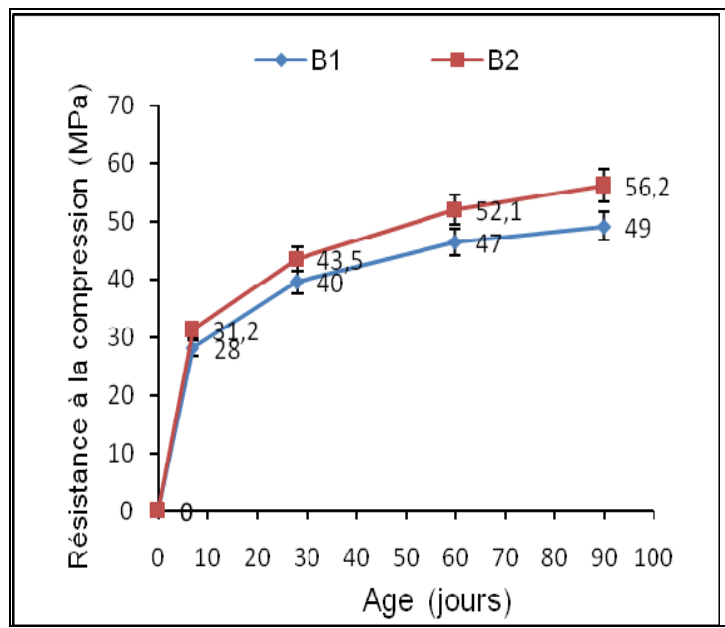

Figure 2. Résistances à la compression des différents bétons.

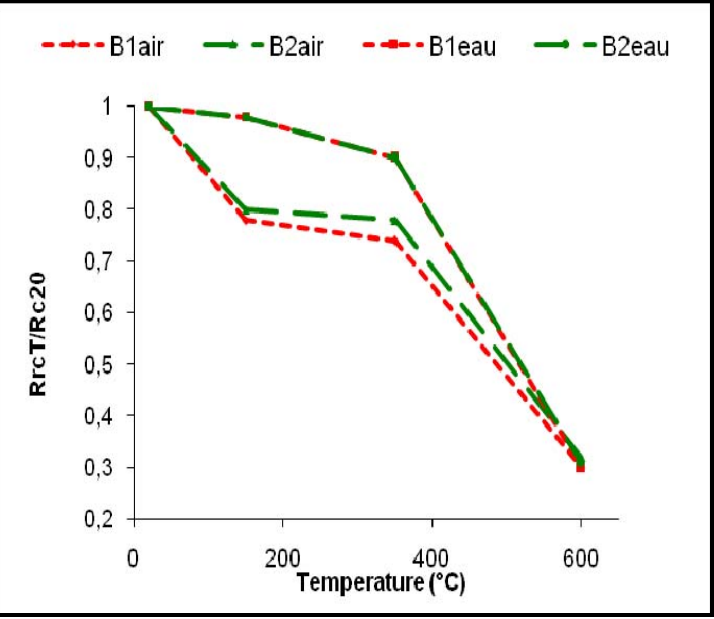

Figure 3. Résistance résiduelle Rcr en fonction de la température.

\section{Conclusions}

À la lecture des résultats donnés dans ce travail, les conclusions sont les suivantes:

a) Un effet bénéfique des SD sur le comportement mécanique du béton B2, à température ambiante et après chauffage. Les DS peuvent partiellement remplacer le sable, en particulier le sable fin, utilisé dans la fabrication du béton.

b) Les effets des températures élevées sur la résistance résiduelle du béton sont plus faibles lorsque le béton est soumis à un choc thermique dans l'air que dans l'eau. 
Mediterranean rocky coasts:

Features, processes, evolution and problems

c) Les bétons chauffés à une température de $600^{\circ} \mathrm{C}$ et soumis à différents traitements de refroidissement ont la même résistance résiduelle. Probablement, ceci est dû au fait qu’à cette température toute l'eau dans le béton a disparu.

\section{Références bibliographiques}

BEN ABDELGHANI F., MAHEREZI W., BOUTOUIL M. (2014). Caractérisation géotechnique des sédiments de dragage marins en vue de leur valorisation en techniques routières. Déchets sciences et techniques, $\mathrm{n}^{\circ}$ 66, pp. 4-13.

http://dx.doi.org/10.4267/dechets-sciences-techniques.269

EN 192-1-2. (2004). Design of concrete structures. Part 1-2: General rules. Structural fire design. European standard, Eurocode 2.

GAO Y.-F., WANG L.-Y., WAN Y.-K., LI B., HE J. (2017). Laboratory study on cement admixed marine silt bricks. Journal Marine Georesources \& Geotechnology, Vol. 35, Issue 2, pp. 186-192.http://dx.doi.org/10.1080/1064119X.2015.1128500

KAZI AOUAL-BENSLAFA F., KERDAL D., SEMCHA A., KAMECHEL Z. E. A. (2011). Contribution à l'étude des risques d'endommagement du revêtement en béton des tunnels soumis aux hautes températures. Revue Tunnels et Espace Souterrain, AFTES, n 223 pp. 85-92.

KAZI AOUAL-BENSLAFA F., KERDAL D., MEKERTA B., SEMCHA A. (2014). The use of dredged sediments as sand in the mortars for tunnel lining and for environmental protection. Arabian Journal for Science and for Engineering, Vol. 39, Issue 4, pp. 2483-2493. http://link.springer.com/article/10.1007\%2Fs13369-013-0805-9

KAZI AOUAL-BENSLAFA F., KERDAL D., AMEUR M., MEKERTA B., SEMCHA A. (2015). Durability of mortars made with dredged sediments. Procedia Engineering, Volume 118, pp. 240-250. http://dx.doi.org/10.1016/j.proeng.2015.08.423

LIMEIRA J., ETXEBERRIA M., AGULLÓ L., MOLINA D. (2011). Mechanical and durability properties of concrete made with dredged marine sand, Construction and Building Materials, Vol. 25, pp. 4165-4174. doi:10.1016/J.CONBUILDMAT.2011.04.053

LUO X., SUN W., CHAN Y. N. S. (2000). Effect of heating and cooling regimes on residual strength and microstructure of normal strength and high-performance concrete. Cement and Concrete Research, Vol. 30 (3), pp. 379-383. doi: 10.1016/S00088846(99)00264-1

PIARC-AIPCR. (2007). Design criteria for structure resistance to fire. Technical report 2007 05.16.B, Resistance to fire of structures, Chapter 9.2, The Road tunnels manual.

ZAMBON A., SAIYOURI N., SBARTAÏ Z.M., DUPLAINE H. (2015). Formulation et comportement d'un béton allégé à base de sédiments marins. Rencontres Universitaires de Génie Civil, Mai 2015, 8p Bayonne, France. hal-01167680

ZHANG B. (2011). Effects of moisture evaporation (weight loss) on fracture properties of high performance concrete subjected to high temperatures. Fire Safety Journal 46 (8) pp. 543-549. doi:10.1016/j.firesaf.2011.07.010 